\title{
SAN JORGE EN LA HIMNOGRAFÍA BIZANTINA: EL HIMNO 66 DE ROMANO MELODO
}

\author{
MIRIAM URBANO-RUIZ \\ Universidad de Granada \\ urmiriam@ugr.es \\ ORCID: 0000-0003-1194-2100
}

\section{RESUMEN}

El himno surge en las civilizaciones primitivas dentro de rituales de culto a las deidades paganas como una especie de poesía sagrada. La introducción del cristianismo marcará definitivamente la remodelación de los himnos griegos, que cuentan con, además de una clara ascendencia clásica, el influjo de la tradición semítica. San Jorge es uno de los retratos de relevancia en el campo de los santos denominados militares y además cuenta con una larga tradición en Occidente y Oriente. Es un santo muy estudiado, pero no por ello carece de interés la revisión de aquellos textos que, aun gozando de un carácter distinto al de los textos hagiográficos principales, aportan datos a su figura y culto. En este trabajo se lleva a cabo la contextualización y un breve análisis de un himno a san Jorge atribuido a Romano Melodo ( $\left.n^{-} 66\right)$, haciendo especial énfasis en la estructura formal y narratológica del texto, de acuerdo con las posibles fuentes hagiográficas.

PALABRAS CLAVE: Himnografía, literatura bizantina, tradición, san Jorge.

\section{SAINT GEORGE IN THE BYZANTINISTIC HYMNOGRAPHY: THE HYMN 66 OF ROMANOS THE MELODIST}

\section{ABSTRACT}

Hymnography finds its origins in cult rituals of pagan civilizations as a kind of sacred poetry. The introduction of Christianity will definitely mark a new era in Greek hymns, comprising not only a clearly classical ancestry, but also influence from Semitic tradition. Saint George is one of the main characters in the sphere of warrior saints, having as well a very long tradition in the West and East. Although he is a much-studied saint, it is noteworthy the revision of those texts, as they provide us with information about his figure and cult. In this essay, a Saint George's hymn attributed to Romanos the Melodist (№ 66) is contextualized and succinctly analysed, with special attention to its formal and narratological structure according to its possible hagiographic sources.

KEYWORDS: Hymnography, Byzantine literature, Tradition, Saint George.

\section{EL HIMNO BIZANTINO}

La cuestión del surgimiento de la himnografía cristiana es un tema muy debatido debido a la falta de fuentes documentales. En etapa bizantina, la evolución del himno es controvertida. Hoy en día se establece una división tripartita de las fases de desarrollo de la himnografía. 
De los tres periodos que se señalan, el primero consiste en una fase de preparación donde la producción se centra en lo que se conoce como tropárion; el segundo, en lo que se considera la etapa de apogeo de la himnografía bizantina, cuya producción se ve centrada en la creación del denominado kontákion; y el tercero, en la creación del kanốn tras la desaparición de los kontákia (McGuckin 2008: 641-675).

El kontákion es un tipo de himno que se desarrolló con gran influencia siríaca $^{1}$ en el transcurso del s. v y que tuvo su máximo apogeo en el s. VI. Este tipo de composición se estructura en tres partes principales; proemio, estribillo y estancias (D'Aiuto 2004: 271, Hörandner 2008: 896, McGuckin 2008: 650-651).

Los himnos bizantinos estaban destinados a cantarse en la liturgia, obedeciendo a una función exclusivamente religiosa (Rotolo 1993: 480). La métrica del kontákion es acentual y sigue un patrón definido que se conoce como heirmós. Este es la melodía base del himno que se presenta en la primera estancia y sirve de modelo para toda la composición. Sus características principales son el isosilabismo y la homotonía. Además, estos himnos estaban compuestos con vistas a su canto y recitación según los ocho tonos de la música bizantina, descritos en el trabajo de Wellesz (1961). En primer lugar, los cuatro

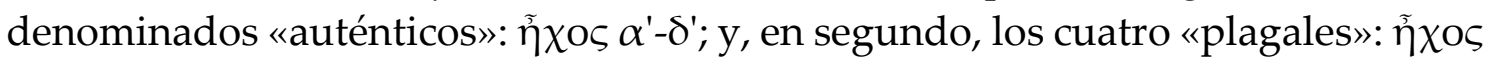
$\pi \lambda . \alpha^{\prime}-\delta^{\prime}$ (D'Aiuto 2004: 279, Wellesz 1961: 300-303).

Según Signes Codoñer (2019: 279), es gracias al género himnográfico que aparecen estos nuevos patrones rítmico-métricos, indiferentes a la métrica clásica, de carácter cuantitativo. En este ámbito juega un importantísimo papel el himnógrafo Romano Melodo, sirio natural de Emesa, que vivió a caballo entre los ss. V y VI. ${ }^{2}$ Es de hecho a este autor a quien se le debe el perfeccionamiento del género, así como la entrada en su etapa de máximo esplendor.

\section{ROMANO MELODO. VIDA Y OBRA}

Hasta finales del siglo pasado se conocían relativamente pocos datos acerca de este himnógrafo. En el Sinaxario Constantinopolitano (cols. 95-96) se nos transmite un breve resumen de la vida del himnógrafo que nos cuenta que Romano era originario de Emesa, ${ }^{3}$ Siria, y que, tras haber sido diácono de la iglesia de Dios conocida como «de la Resurrección» en Beirut, llegó a Constantinopla en tiempos

\footnotetext{
${ }^{1}$ La afinidad del kontákion con la poesía litúrgica siríaca queda de manifiesto y puede incluso verse como una transposición en lengua griega de un género que fue formado y evolucionó casi por completo en su lengua siríaca natal. Sus principales representantes son los géneros del mêmrâ, el mâdrâshâ y la sôgîthâ, según Grosdidier (1977: 16-17).

2 Su obra hoy en día comprende un total de 89 piezas himnódicas, de las cuales únicamente 59 se consideran genuinas sin duda alguna (vid. Maas-Trypanis 1963, 1970).

${ }^{3}$ No se conocen los datos exactos acerca del nacimiento del himnógrafo, pero generalmente se sitúa en torno al año 485 en el seno de una familia judía.
} 
del emperador Anastasio ${ }^{4}$ y se instaló en el templo de la Madre de Dios ${ }^{5}$ en el barrio de Kýros. Allí fue donde recibió el don de componer kontákia de parte de la Virgen, ${ }^{6}$ que se le apareció en sueños en el día del nacimiento de Cristo y le dio un pedazo de papiro para que lo comiera. Súbitamente se despertó, subió al ambón y comenzó a cantar su Himno a la Natividad. Este talento, de procedencia divina, lo acompañaría a lo largo de toda su vida, que dedicó a la creación de este tipo de poemas. Murió en Constantinopla y fue enterrado en la misma iglesia donde había ejercido su ministerio. ${ }^{7}$

La obra de Romano había permanecido casi totalmente desconocida hasta la publicación de los Analecta Sacra por parte de J. B. Pitra (1876), donde se hallaban veintinueve de los himnos de Romano. Fue ya K. Krumbacher (1911) quien se propuso llevar a cabo una edición de todos sus himnos, tarea que quedaría en manos de su alumno, P. Maas y de C. A. Trypanis (1963), y que sería retomada más tarde por J. Grosdidier de Matons (1977).

De las mil piezas que la tradición atribuye a Romano, ochenta y nueve han llegado hasta nuestros días, y no todas se consideran genuinas. Estas están claramente divididas por su temática: treinta y cuatro tratan sobre Cristo, siendo las principales de su obra, mientras que el resto encuentra su inspiración en figuras diversas.

\subsection{Himnos a san Jorge atribuidos a Romano Melodo}

En cuanto a los himnos a san Jorge de Romano, tanto Krumbacher (1911) como Maas y Trypanis (1970) cuentan con ediciones de estos. Las piezas hímnicas editadas por Krumbacher fueron recogidas y publicadas tras su muerte, gracias

\footnotetext{
${ }^{4}$ Se sabe que en Bizancio hubo dos emperadores que llevaron por nombre Anastasio. Ninguna de las fuentes que hablan de Romano especifica cuál de los dos emperadores homónimos reina en el momento de su traslado a Constantinopla. La necesidad de concretar cuál de los dos fue quien reinó en aquellos momentos se hace evidente si se tiene en cuenta que de su cronología depende, no solo la fecha en la que Romano vivió o la de la composición de sus himnos, sino también la del momento de apogeo máximo del kontákion tras la evolución experimentada por parte del género hímnico. De los dos emperadores mencionados, Anastasio I gobernó entre los años 491 y 518; y Anastasio II, entre 713-715. Bousquet (1900) dice que, tras el descubrimiento de Romano por parte de Pitra, una de las cuestiones más importantes que queda en el aire es si vivió en tiempos del primero o del segundo. Mientras que Krumbacher se inclinó en su momento en favor del segundo de los emperadores, hoy en día la opinión no es la misma: por ejemplo, Pitra (AS I: XXVI-XXVIII) se decanta por Anastasio I, Grosdidier (1977: 175-178) parece aceptar esta teoría, así como Maas y Trypanis (1963: XVI) y Mulard (2016: 11).

${ }^{5}$ La iglesia formaba parte de un monasterio homónimo del barrio, nombrado así por el prefecto Ciro de Panópolis, quien murió en el año 45. Este templo dedicado a la Virgen fue la primera de las iglesias marianas de Constantinopla (Arentzen 2017: 191).

${ }^{6}$ Es famosa la miniatura del cod. Vat. gr. 1613, que representa este episodio en el que Romano obtiene el don de la composición de himnos por haber comido un papiro que la Virgen le entrega en sueños.

${ }^{7}$ Romano murió con toda seguridad un 1 de octubre, ca. 555.
} 
A. Ehrhard, en el estudio Der heilige Georg in der griechischen Überlieferung (1911: 84-95). La edición del texto de Maas y Trypanis se encuentra en el volumen Sancti Romani Melodi cantica. Cantica dubia (1970: 45-58).

Únicamente aportan el texto de los himnos a san Jorge dos manuscritos: ${ }^{8}$ el primero de ellos es el cod. Mosquensis Synod. 437 (s. XII), y el segundo el cod. Patmiacus 213 (s. XI). La tradición manuscrita para ambas ediciones es la misma; beberán de los códices anteriormente mencionados tanto Krumbacher como Maas y Trypanis.

El primero de los himnos $-\mathrm{n}^{\mathrm{o}} 66$ - tiene como fuente principal una vida de san Jorge atribuida a Pasícrates. Maas y Trypanis (1970: 193) ponen en duda la autoría de Romano argumentando que la fecha de composición de tal documento se sitúa en el s. VII, pero, teniendo en cuenta que la versión interpolada estaba difundida ya en el s. VI (Krumbacher 1911: 290), no se puede descartar la autoría de Romano con este argumento.

El segundo himno - no $67-$ también ha generado cierto escepticismo entre los estudiosos; hoy en día se considera espurio sin duda alguna, como se puede observar gracias al epíteto del acróstico del texto y algunos elementos de carácter lingüístico o retórico, que probablemente no serían de la elección del himnógrafo (Maas y Trypanis 1970: 194).

\subsubsection{Breve anotación sobre el santo}

San Jorge es una de las figuras centrales del cristianismo y ocupa un lugar privilegiado en el mundo eclesiástico y el sistema cultural. Los textos acerca de su persona conforman una masa, cuanto menos, confusa. La primera edición de su tradición hagiográfica se halla en los Acta Sanctorum y pertenece a D. Papebroch, J. Bolland y G. Henschen (1675), pero es a principios del s. XX cuando la cuestión llega a su momento máximo de auge, con las ediciones a cargo de Delehaye (1909), Krumbacher (1911) y J. B. Aufhauser (1913), revisadas y ampliadas, como resultado.

El testimonio más antiguo de la Passio de san Jorge se halla en el palimpsesto de Viena de manera muy fragmentaria y fue editado por D. Detlefsen (1858), F. Vetter (1896) y Krumbacher (1911). Esta redacción de la vida de san Jorge se nombra como Dadianustypus, ya que en ella es Dadiano el rey imperante y antagonista del joven mártir. Dado que es un relato de corte oriental con muchos elementos de carácter fabuloso acabó siendo condenado entre los textos apócrifos por el Papa Gelasio I en el año 494 (Casas Olea 2018).

La segunda de las redacciones es la llamada Diokletiantypus. Aunque esta versión de la Passio no cuenta ya con los motivos fantásticos del Dadianustypus, sí que recoge relatos típicos hagiográficos, como la genealogía y la infancia del

\footnotetext{
${ }^{8}$ Maas y Trypanis (1963: XXV-XXVIII; 1970: XIV-XV) dividen en dos grupos los manuscritos que transmiten la obra del himnógrafo: por un lado, los códices primarios, y por otro, los secundarios. Tanto el Mosquensis como el Patmiacus pertenecen al primer bloque mencionado.
} 
santo, que confieren historicidad al relato. Dentro de esta variante es posible distinguir también diferentes redacciones. La más simple es la llamada por Krumbacher Normaltext (Krumbacher 1911: 41-51). No obstante, hay otra variante la cual probablemente se gestó en el mismo tiempo e incluye el relato de la infancia y la juventud (Krumbacher 1911: 51-58); se conoce como interpolierte Normaltext.

Casas Olea (2018) ofrece una distribución del dossier hagiográfico del santo según las diferentes tipologías de texto. Así, hace notar que además de las Passiones $^{9}$ - agrupadas en las dos variantes ya mencionadas (BHG ${ }^{10}$ 670-677) - , la tradición hagiográfica de san Jorge cuenta con una serie de Laudationes, donde tienen cabida himnos compuestos por autores como Romano Melodo o Andrés de Creta (BHG 681-686). A estas se adhiere la colección de Miracula (BHG 687-691), en los cuales se narra el episodio del dragón, que es el más difundido (BHG 687), pero también otros como el de De bobus Theopisti (BHG 689) o el De columna viduae (BHG 691).

\subsubsection{Primer himno a san Jorge}

El himno 66 de Romano Melodo representa de manera muy clara el esquema formal del kontákion. En primer lugar, cuenta con un proemio de siete versos, siendo el último de ellos uno de los dos refranes que se alternan en este himno. ${ }^{11}$

A continuación, se presentan las veinticuatro estancias - también de siete versos - que, sucediéndose, conforman el poema. Estas alternan en su cierre dos estribillos diferentes: el ya introducido en el proemio y otro que aparece por primera vez en la quinta estancia. ${ }^{12}$

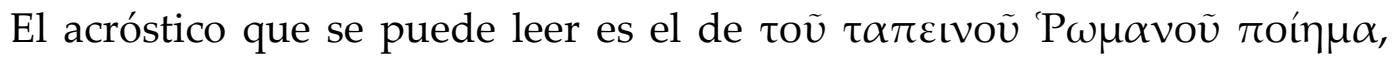
formado por la primera letra de cada una de las estancias, sin tener en cuenta el proemio. Los heirmoí13 a los que se ajustan los versos de Melodo son dos. El

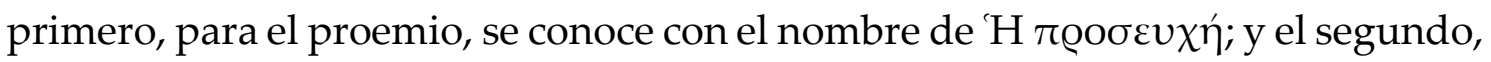

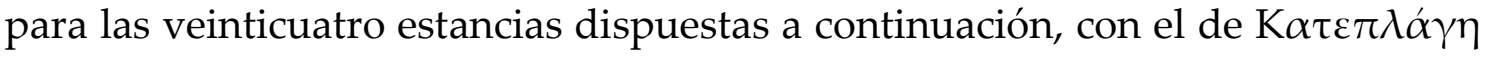

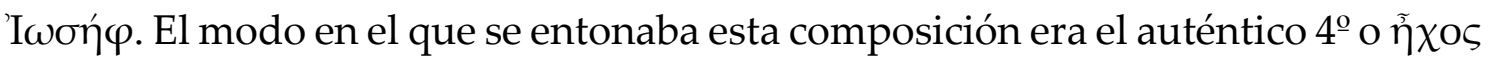
$\delta '$.

La estructura narrativa del Himno a san Jorge se divide en cuatro partes. La primera es el proemio. En ella se hace una introducción al tema que se va a tratar a continuación a lo largo de la composición. La segunda está constituida por las

\footnotetext{
9 Precisamente las Passiones constituyen la base sobre la que Romano construye la secuencia narrativa del himno. También aparecen en BHG una serie de Vitae et martiria (BHG 678-679) y una Conceptio et Nativitas (BHG 680) del santo.

10 i. e. Bibliotheca Hagiographica Graeca.

11 Vid. Maas-Trypanis (1970: 45).

12 Vid. Maas-Trypanis (1970: 47).

13 Para más exactitud en los patrones rítmicos de los versos de este himno y sus variantes, vid. Maas-Trypanis (1963: 511 ss., 1970: 210 ss.).
} 
cuatro primeras estancias, que tienen un claro contenido de carácter doxológico. A continuación, la tercera parte del poema son las estancias cinco a veintitrés, que constituyen el núcleo del himno; cada una de ellas narra un episodio de la hagiografía. Finalmente, la última parte, que es la estancia final. ${ }^{14}$ De esta manera se cierra el himno con una breve oración y se hace una petición para el pueblo y el himnógrafo.

El tema central del himno es la narración de la vida de san Jorge. En ellas se habla de los padres del santo, Gerontio y Policronia (66.ع.1-3); se narra la juventud del santo y se citan los cargos que ostentó (66.5.1-3); Jorge aconseja a su padre, quien a la vejez se convierte a la fe cristiana (66.ๆ.1-7); destruye los ídolos (66.ı.3); es llevado ante el emperador Diocleciano, quien está furioso por ello y lo martiriza (66.ı $\alpha .1-4)$; pero se niega a sacrificar a los ídolos tras el interrogatorio que mantiene con el emperador (66.เ $\gamma .3-7)$; se salva mediante una oración a Dios pese a la magnitud de todos los tormentos (66.lع.1-7); convence a la emperatriz Alejandra de convertirse a la fe cristiana, por lo que posteriormente muere

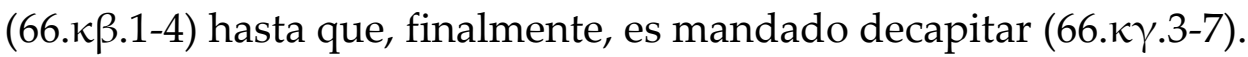

En cuanto al registro lingüístico que presenta la pieza himnográfica, cabe hacer notar que Romano es buen conocedor de la gramática y prosodia griegas, por lo que apenas hay excepciones a lo que entendemos como regla en este himno dedicado a san Jorge. Además, el léxico que se observa es en general relativo al ámbito clásico. Llaman la atención, entre otros, elementos como los préstamos y

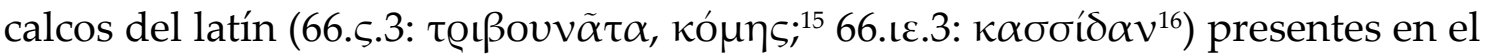
texto, la simplificación de paradigmas verbales que se observa en $66 . \delta .2\left(\delta\left\llcorner\delta \tilde{\omega} v,{ }^{17}\right.\right.$ de $\delta i ́ \delta \omega, \delta\llcorner\delta \tilde{\omega})$, la confusión entre activa y pasiva en el caso de algunos aoristos

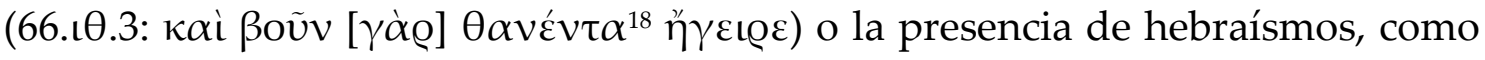

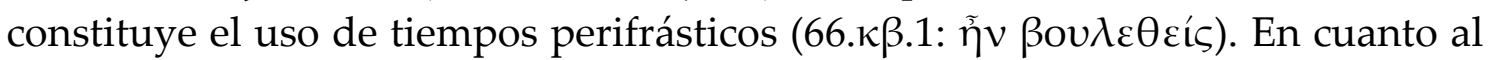
uso excesivo o defectivo del artículo o a las - pocas - variaciones en la acentuación, son fenómenos que atañen al ámbito de la métrica o ritmo acentual de la pieza himnódica (Mitsakis 1965, 1967).

\section{CONCLUSIONES}

Se pueden extraer dos conclusiones principales a partir de los datos expuestos. La primera es que por la propia secuencia narrativa que sigue el himnógrafo - la narración de la infancia y juventud del santo, la aparición de personajes como sus padres o la conversión la emperatriz Alejandra y su muerte mientras

\footnotetext{
14 Vid. Maas-Trypanis (1970: 52).

${ }^{15}$ Cf. lat. tribunatum (pl. tribunata), comes.

${ }^{16}$ Cf. cassis. Sobre la formación de $\kappa \alpha \sigma \sigma i ́ \delta \alpha v$ : Mitsakis 1965: 187, 1967: 28. También vid. Trapp,

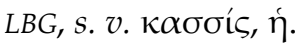

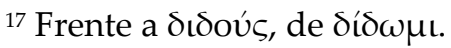

18 En lugar de la voz activa $\theta \alpha$ vóvt $\alpha$.
} 
se desarrolla el martirio - se determina que la fuente principal de la que se toma la estructura narrativa del poema es la variante de la vida de san Jorge conocida como Diokletiantypus. Romano debió de seleccionar la redacción interpolada de esta variante como fuente para el himno precisamente por estar dispuesta para un auditorio no culto, al igual que lo estaban sus poemas. La elección de la variante, dada por una motivación popular y de novedad sirve, además, para justificar la afirmación de la atribución de la autoría de Romano a este himno. Pese a que Maas y Trypanis (1970: 173) la ponen en duda argumentando que la redacción pertenece al s. VII, es asumible la propuesta de Krumbacher, que afirma que la redacción del Diokletiantypus y su versión interpolada se localiza en el s. VI.

La segunda es que la situación de la lengua en Bizancio es una de las piezas clave para comprender los hechos lingüísticos que se observan en el texto - el cual, por razones de brevedad, hemos dejado fuera del trabajo. El Imperio se hallaba en situación de diglosia con dos variantes lingüísticas funcionalmente complementarias, una popular y otra literaria aticista, a lo que se añadía la imposición del latín como lengua oficial. Si además se tiene en cuenta el bilingüismo presente en las comunidades de Asia Menor en las que se hablaba griego como segundo idioma, se comprende el porqué de la complicada situación en que se encontraba la lengua griega de la época (Horrocks 2010: 189-368). Todo esto justifica los hechos lingüísticos mencionados en el epígrafe anterior.

En fin, la lengua del himnógrafo Romano Melodo intenta acercarse al pueblo, mostrándose sencilla y fácil de comprender y adaptándose a unas necesidades nuevas, propias de un público nuevo. Esto no implica que Romano haga un mal uso de la lengua griega a propósito, o que sea desconocedor de las normas, sino todo lo contrario: sabe a quién se dirige y conoce sus necesidades.

\section{BIBLIOGRAFÍA}

Arentzen, TH. (2017), The Virgin in Song: Mary and the poetry of Romanos the Melodist, Philadelphia, University of Pennsylvania.

AufHAuser, J. (1913), Miracula Sancti Georgii, Leipzig, Teubner.

BousQueT, R. (1900), «Le culte de saint Romain le Mélode dans l’Église grecque et l'Église arménienne», Échos d'Orient, 6(3), 339-342.

CASAs OleA, M. (marzo de 2018), «Literacy and Orality in Traditions and Cults of Saint George», en Heroic Saints or Holy Heroes: the Blending of Epic and Hagiography between Orality and Literacy in the Popular Religion of Eastern Christianity, European Academy of Religion First Annual Conference.

D'AIUTO, F. (2004), «L'innografia», en Lo spazio letterario del Medioevo. III le culture circostanti, Capaldo, M., Cardini, F., Cavallo, G. y Scarcia Amoretti, B. (eds.), vol. I, Roma, Salerno, 257-300.

Delehaye, H. (1902), Synaxarium Ecclesiae Constantinopolitanae e codice Sirmondiano. Propylaeum ad Acta Sanctorum Novembris, Bruselas, Société des Bollandistes, cols. 95-96. 
DeleHAYE, H. (1909), «Saint Georges», en Les légendes grecques des saints militaires, París, Alphonse Picard et Fils, 45-76.

DeTLEFSEN, D. (1858), Über einen griechischen Palimpsest der K. K. Hofbibliothek mit Bruchstücken einer Legende vom heiligen Georg, Viena, Akademie der Wissenschaften.

GROSDIDIER DE MATONS, J. (1977), Romanos le Mélode et les origines de la poésie réligieuse à Byzance, París, Beauchesne.

HALKIN, F. (1957), Bibliotheca hagiographica graeca, 3 vols., Bruselas, Societé des Bollandistes.

HÖRANDNER, W. (2008), «Poetry and romances», en The Oxford Handbook of Byzantine Studies, Jeffreys, E., Haldon, J. y Cormack, R. (eds.), Nueva York, Oxford University Press, 896.

HORROCKS, G. (2010), Greek. A History of the Language and its Speakers, Malden MA-Oxford, John Wiley \& Sons, 189-368.

KRUMBACHER, K. (1911), Der heilige Georg in der griechischen Überlieferung, Múnich, Akademie der Wissenchaften.

MAAS, P. y TRYPANIS, C. A. (1963), Sancti Romani Melodi Cantica. Cantica genuina, Oxford, Oxford University Press.

MAAS, P. y TrypanIS, C. A. (1970), Sancti Romani Melodi Cantica. Cantica dubia, Berlín, De Gruyter.

MCGuKIN, J. A. (2008), «Poetry and Hymnography (2): The Greek World», en The Oxford Handbook of Early Christian Studies, Harvey, S. A. y Hunter, D. G. (eds.), Nueva York, Oxford University Press, 641-657.

MITSAKIS, K. (1965), «The vocabulary of Romanos the Melodist», Glotta, 43, 171-197.

MITSAKIS, K. (1967), The language of Romanos the Melodist, Múnich, C. H. Beck'sche.

MulARD, CH. (2016), La pensée symbolique de Romanos le Mélode, Turnhout, Brepols.

PAPEBROCH, D., Bolland, J. y Henschen, G. (eds.) (1675), Acta Sanctorum Aprilis collecta, digesta, illustrata, a Godefrido Henschenio et Daniele Papebrochio e Societate Iesu. Tomus III quo ultimi IX dies continentur. Præmittitur Exegesis Præliminaris Diatribam de tribus Dagobertis olim æditam innovans et stabiliens. Subiunguntur Acta Græca, ad eosdem dies pertinentia, Antwerp, Chadwyck-Healey.

PITRA, J. B. (1876), Analecta Sacra Spicilegio Solesmensi Parata I, París.

Rotolo, V. (1993), «La poesia», en Lo spazio letterario della Grecia antica, Cambiano, G., Canfora, L. y Lanza, D. (eds.), vol. II, Roma, Salerno, 478-484.

Signes Codoñer, J. (2019), Breve guía de la literatura griega desde Hesíodo hasta Pletón, Madrid, Cátedra.

VETTER, F. (1896), Der heilige Georg des Reinbot von Durne: mit einer Einleitung über die Legende und das Gedicht, Halle, Niemeyer.

Wellesz, E. (1961), A History of Byzantine Music and Hymnography, Oxford, Clarendon Press. 